\title{
Análisis de ítems y evidencias de fiabilidad de la Escala sobre Representación Cognitiva de la Enfermedad (ERCE)
}

\author{
Macarena De los Santos-Roig* y Cristino Pérez-Meléndez
}

Universidad de Granada. Facultad de Psicología

\begin{abstract}
Resumen: La Escala sobre Representación Cognitiva de la Enfermedad, ERCE (De los Santos-Roig, 2009) ha sido elaborada para evaluar las creencias de los pacientes sobre su enfermedad. El modelo de Sentido Común, donde se inserta el constructo, ha sido puesto a prueba en numerosas ocasiones, encontrándose evidencia sobre la importancia de la Representación Cognitiva en los procesos de adaptación a la enfermedad (Hagger y Orbell, 2003; Leventhal et al., 1997). Su medida en nuestro ámbito cultural es necesaria, tanto desde un punto de vista teórico, como desde una perspectiva aplicada. Lo que aquí se muestra es la continuación a un trabajo previo (De los Santos-Roig, 2009; De los Santos-Roig y Pérez-Meléndez, 2013) donde se concretó la definición semántica del constructo RCE y las Tablas de Especificaciones del Test y de los Ítems, que finalmente culminó con la presentación de los primeros ítems construidos. Siguiendo con lo establecido en los estándares (AERA, APA y NCME, 1999), se muestran ahora los resultados del análisis estadístico de los ítems correspondientes a las cinco escalas que componen la ERCE, así como los obtenidos sobre sus evidencias de fiabilidad. Al final, se concluye sobre la idoneidad de los ítems que conforman la escala.

Palabras clave: Representación cognitiva de la enfermedad; Escala Erce; Análisis de ítems; Análisis factorial; Fiabilidad.
\end{abstract}

\section{Introducción}

Existen algunos trabajos en nuestro país sobre instrumentos adaptados para medir la llamada Representación de la Enfermedad, RE (Beléndez-Vázquez, Bermejo-Alegría y GarcíaAyala, 2005; Van der Hofstadt y Rodríguez-Marín, 1997); un constructo inserto en el Modelo de Sentido Común sobre la enfermedad desarrollado por Leventhal y otros, para dar respuestas sobre el afrontamiento y la adaptación de los pacientes en los procesos de salud-enfermedad. Desde esta teoría, se asume que la RE actúa a modo de esquema que afecta bidireccionalmente al modo de afrontar la enfermedad, así como al estatus del paciente (Leventhal et al., 1977; Leventhal, Brissette y Leventhal, 2003). Multitud de trabajos han dado cuenta de estas relaciones así como de su influencia en la recuperación o la vuelta al trabajo, el autocuidado, la adherencia al tratamiento, así como el ajuste psicológico, no sólo en una, sino a través de multitud de enfermedades (Broadbent, Donkin y Stroh, 2011; Hagger y Orbell, 2003; Hoving, Van der Meer, Volkova y Frings-Dresen, 2010; McSharry, Moss-Morris y Kendrick, 2011).

Existen instrumentos en nuestro país, como se indicaba anteriormente, desarrollados para evaluar el constructo RE (el Illness Perception Questionnaire, IPQ-versión española de Beléndez-Vázquez et al., 2005; el CCRE que es la versión española del IMIQ-Implicit Models of Illness Questionnaire- de Van der

* Dirección para correspondencia [Correspondence address]:

Macarena De los Santos-Roig. Departamento de Metodología de las Ciencias del Comportamiento. Facultad de Psicología. Universidad de Granada. Facultad de Psicología. Campus Cartuja. 18071. Granada (España).E-mail: dlsantos@ugr.es
Title: Items analysis and reliability evidences of ERCE scale.

Abstract: ERCE Scale (De los Santos-Roig, 2009) was developed to assess patients' beliefs about illness. They are assumed to be related to coping and health outcomes in Leventhal's Common Sense Model (Hagger and Orbell, 2003; Leventhal et al., 1997). This model has been proved empirically and evidence about the relevant role of Cognitive Illness Representation has been found. The need of an instrument able to assess Illness Representation in our cultural setting is highlighted, because of its theoretical importance for the model but for the measure and assessment of physically ill patients, from an applied perspective. The aim of this work is to present the results about item analysis, factorial analysis and reliability evidences of ERCE and its five sub-scales scores. This will be presented as a continuation of first steps done (semantic definition of construct, items and test specifications, preliminary items, etc.) showed in other papers (De los Santos-Roig, 2009; De los Santos-Roig y Pérez-Meléndez, 2013). We conclude about the adequacy of items.

Key words: Cognitive illness representation; Common Sense model; Erce scale; Reliability; Items analysis.

Hofstadt y Rodríguez-Marín, 1997). Sin embargo, éstos muestran una serie de limitaciones (como la escasa definición de los componentes, la traducción de los ítems y la validación a través únicamente del análisis factorial, el uso de muestras de participantes sanos en lugar de enfermos, etc.), que hacen insatisfactorios, en nuestra opinión, los resultados de la medición. De ahí surge inicialmente la necesidad de elaboración de una nueva escala, la escala ERCE (De los Santos-Roig, 2009), formada por cinco sub-escalas que versan sobre sus componentes "cognitivos" (a saber, la identidad o síntomas característicos de la enfermedad, causas de la misma, control sobre ella, consecuencias en distintas facetas de la vida de la persona y curso temporal). No se aborda en esta escala, por tanto, la parte "emocional" de la representación, contemplada también en el modelo pero cuya definición y concreción teórica hasta ahora no ha resultado suficiente, desde nuestro punto de vista, para que pueda iniciarse el proceso de medición.

En un trabajo previo a éste (De los Santos-Roig y PérezMeléndez, 2013) se presentó la justificación teórica, la definición semántica del constructo, las Tablas de Especificaciones del Test y de los Ítems de la escala, se mostraron los primeros elementos desarrollados y su evaluación por parte de expertos (juicio sobre los ítems), así como la puesta a prueba con pequeñas muestras de pacientes. Una vez concluido sobre este análisis de tipo cualitativo, se realizó el análisis sobre la calidad métrica de los mismos.

El presente trabajo tiene como objetivo mostrar los resultados del análisis de ítems, del análisis factorial exploratorio y las evidencias de fiabilidad, así como comentar las conclusiones que se alcanzaron. No debe olvidarse, sin embar- 
go, que se trata de un trabajo basado en el anterior y que es continuo a éste, ni dejar de considerarse que ambos forman un todo relativo al desarrollo de la Escala ERCE.

$\mathrm{El}$ análisis de ítems se va a presentar desde una perspectiva clásica (descriptivos, homogeneidad y discriminación). Después se procederá a mostrar la estructura factorial de las escalas de consecuencias y control/cura por separado, ya que son escalas independientes no sumativas, en las que lo que interesa comprobar es si los ítems se agrupan reproduciendo las definiciones (componente y subcomponentes). El objetivo de este análisis factorial no es más que el obtener evidencias de validez de contenido o, dicho de otro modo, si los ítems representan el contenido a partir del cual se han definido estos dos componentes del constructo. Para el resto no se ha contemplado este tipo de análisis, dada la peculiar naturaleza de los ítems que las componen. Por último se mostrarán las evidencias de fiabilidad que se obtuvieron para todas las escalas.

\section{Método}

\section{Participantes}

Para el estudio se accedió a la población de pacientes diabéticos que acudían al Hospital de Día del Servicio de Endocrinología del Hospital Universitario San Cecilio (Granada). Se obtuvo una muestra clínica incidental, compuesta por aquellos pacientes ingresados durante los meses de Febrero de 2008 a Marzo de 2009 que voluntariamente quisieron participar y que cumplían ciertos requisitos para su inclusión; que fueran Tipo 1 (menos frecuentes en la población de diabéticos), sin complicaciones, insulinodependientes, sin otras patologías o psicopatologías, mayores de edad y con al menos un año de evolución de la enfermedad.

Finalmente, la muestra se conformó de un total de 136 diabéticos Tipo 1 , de los que un $51.5 \%$ eran hombres. La edad de los participantes estuvo comprendida entre los 18 y los 68 años, con una media de $34.14(D T=9.27)$. Por otro lado, el 36.8\% tenía estudios secundarios y el 34.6\% universitarios. Del total de 136 pacientes, 63 de ellos dieron su conformidad para volver a contestar los cuestionarios en la fase del re-test. De ahí que estos análisis se presenten posteriormente con un tamaño muestral más pequeño.

\section{Instrumentos}

Los participantes cumplimentaron un cuadernillo compuesto por las cinco sub-escalas no sumativas que forman la ERCE (De los Santos-Roig, 2009). La primera es la escala de identidad, que consiste en un listado de 20 síntomas a marcar en caso de presencia, junto a una pregunta sobre la frecuencia de su relación con la diabetes (no, a veces, si). La escala causal consta de otro listado de 7 posibles causas, en este caso atribuibles al origen de la enfermedad (causas medioambientales, psicológicas, biológicas, entre otras), así como 10 situaciones antecedentes a las crisis de hiper/hipoglucemia. Las escalas de consecuencias (16 ítems), control/ cura (12 ítems) y curso (2 ítems), se responden a través de otra escala likert que, para las dos primeras, es de 5 alternativas (cuyas etiquetas varían en grado, desde nada a mucho, en función de la pregunta), y que para los ítems del curso tiene 3 y 4 alternativas, respectivamente.

No hay ningún ítem inverso y la interpretación de las puntuaciones se realizaría de modo que a mayor cantidad de síntomas que el paciente relaciona con la enfermedad, mayor identidad de la misma. Es necesario hacer hincapié en la necesidad de separar la cantidad de síntomas (en número), de la relación de éstos con la diabetes (es decir, de todos los marcados, ¿cuáles se relacionan siempre, a veces o nunca con la diabetes?). Esta distinción ha sido enfatizada por otros autores con objeto de distinguirla de constructos tales como, por ejemplo, la hipocondría (Hagger y Orbell, 2003). Las causas, que se separan en dos bloques; del origen y situacionales, se interpretarían de manera distinta. Las del origen de la enfermedad no serían sumativas (el paciente marca la o las que atribuye al origen de ésta). Las causas situacionales, que provocan crisis de hiper e hipoglucemia, sí podrían sumarse si se quisiera realizar algún tipo de diagnóstico. Así, a mayor cantidad, más situaciones que el paciente reconoce como desencadenantes/causas de malestar. En este trabajo, ambos bloques de causas se van a considerar por separado y nunca de forma sumativa. Por último, las consecuencias, el control/cura y el curso, se interpretan como que a mayores puntuaciones en consecuencias más impacto en la vida del paciente, a menores puntuaciones en control menor percepción de capacidad de control sobre la misma y mayores puntuaciones en curso indicarían una percepción de la enfermedad con tendencia a empeorar con el paso del tiempo y muy duradera.

\section{Procedimiento}

La recogida de datos se llevó a cabo en la sala de educación diabetológica del Hospital de Día y el procedimiento fue igual para todos los participantes durante todo el tiempo que duró la recogida de datos. Hay que indicar que el comité ético del hospital dio su aprobación para la realización del estudio y siempre se pidió al paciente su consentimiento firmado, además de garantizarle la confidencialidad de las respuestas a través del anonimato.

\section{Análisis de datos}

En las escalas de Identidad y Causas de la enfermedad, al tratarse de listados, el análisis de los ítems se centró exclusivamente en la frecuencia de elección. Las respuestas a los ítems de las escalas de consecuencias, control-cura y curso, fueron analizadas descriptivamente y también se estudió su homogeneidad (correlación item-total corregida) así como su capacidad de discriminación. Éste último análisis es, a nuestro juicio, imprescindible y muy informativo, ya que permite comprobar ítem a ítem la capacidad de discriminar entre perfiles ra- 
dicalmente distintos en el modo de representar la enfermedad. En este momento ésta puede parecer una cuestión irrelevante cuya importancia estará, sin duda, en los estudios posteriores sobre la validez de las puntuaciones en la escala. Sin embargo, se ha incluido ya que tenía perfecta cabida desde un punto de vista exploratorio en general y, en particular, desde el análisis de ítems.

Como ahora se verá, también se realizó un análisis factorial exploratorio $(A F E)$ sobre la matriz de correlaciones policóricas utilizando mínimos cuadrados ponderados (WLSM) y rotación Oblimin. El objetivo de este análisis fue el de comprobar si los ítems de las escalas de consecuencias y control/cura reproducían la estructura de sus respectivas definiciones. Se eligió este tipo de rotación ya que se presupone que los sub-componentes no son ortogonales.

Finalmente, en el estudio de las evidencias de fiabilidad, se utilizó el test-retest (y las discrepancias, que más adelante se explicarán) para comprobar la estabilidad de las puntuaciones en el tiempo, así como la consistencia interna mediante el coeficiente alfa de Cronbach en aquellas escalas en que esto fuera posible.

Para todo se usó el programa SPSS (v.15), excepto en el AFE, que fue realizado con el programa MPlus (v.5.1.).

\section{Resultados}

En todas las tablas de resultados, se hace referencia a los ítems mediante una etiqueta. En caso de querer consultar el contenido de un ítem concreto véase el Anexo, donde se presenta la ERCE completa (ítems e instrucciones para las cinco escalas).

Frecuencias de elección de los ítems de las escalas de Identidad y Causas

En la Tablas 1 y 2 aparecen los resultados sobre las frecuencias de respuesta para los elementos de las escalas de Identidad y Causas.

La información obtenida indica cómo todos los síntomas de la escala de identidad han sido elegidos (Tabla 1) y cómo para la mayoría de ellos las respuestas sobre su relación con la enfermedad ha mostrado suficiente variabilidad. Sobre la escala de causas (Tabla 2), los resultados indican que todas (del origen de la enfermedad y situacionales) han sido seleccionadas, aunque algunas son más frecuentemente elegidas que otras.

\section{Resultados sobre los estadísticos básicos, homoge- neidad y discriminación de los ítems de consecuen- cias, control-cura y curso}

Lo que se observa en la Tabla 3 para los ítems de la escala de consecuencias es que todas las opciones de respuesta de la escala tipo Likert han sido elegidas en la totalidad de los ítems, la mayoría de las medias están en torno al valor central, la desviación típica en torno a 1 y los índices de asimetría son significativos en algunos casos (C.Sintomas2, C.Curso2, C.Control2, C.Futuro1, C.Futuro2). En lo que se refiere a la curtosis, aparece significativa en muchos de los ítems, donde se encuentra una distribución platicúrtica.

Tabla 1. Frecuencias de respuesta a los items de la escala de identidad ${ }^{t}$

\begin{tabular}{|c|c|c|c|c|c|}
\hline Síntomas & & Presente & Si & A veces & No \\
\hline 1 & Sudoración & $79.7 \%$ & $69.8 \%$ & $26.4 \%$ & $3.8 \%$ \\
\hline 2 & Temblores & $71.4 \%$ & $82.1 \%$ & $17.9 \%$ & - \\
\hline 3 & Nerviosismo & $51.9 \%$ & $24.6 \%$ & $43.5 \%$ & $31.9 \%$ \\
\hline 4 & Hambre & $68.4 \%$ & $63 \%$ & $31.5 \%$ & $5.4 \%$ \\
\hline 5 & Debilidad & $55.6 \%$ & $62.5 \%$ & $33.3 \%$ & $4.2 \%$ \\
\hline 6 & Palpitaciones & $36.1 \%$ & $58.3 \%$ & $33.3 \%$ & $8.3 \%$ \\
\hline 7 & Ardores & $26.3 \%$ & $20 \%$ & $11.4 \%$ & $68.6 \%$ \\
\hline 8 & Hormigueo & $39.1 \%$ & $76.9 \%$ & $17.3 \%$ & $5.8 \%$ \\
\hline 9 & Nauseas & $18 \%$ & $54.2 \%$ & $25 \%$ & $20.8 \%$ \\
\hline 10 & Dolor de cabeza & $57.1 \%$ & $33.3 \%$ & $46.7 \%$ & $20 \%$ \\
\hline 11 & $\begin{array}{l}\text { Dificultad para hablar } \\
\text { o moverse }\end{array}$ & $27.1 \%$ & $82.9 \%$ & $14.3 \%$ & $2.9 \%$ \\
\hline 12 & Agresividad & $31.6 \%$ & $57.1 \%$ & $35.7 \%$ & $7.1 \%$ \\
\hline 13 & $\begin{array}{l}\text { Problemas para ver } \\
\text { bien }\end{array}$ & $42.9 \%$ & $61.4 \%$ & $28.1 \%$ & $10.5 \%$ \\
\hline 14 & Desorientación & $24.1 \%$ & $83.9 \%$ & $12.9 \%$ & $3.2 \%$ \\
\hline 15 & Mareo & $47.4 \%$ & $72.6 \%$ & $22.6 \%$ & $4.8 \%$ \\
\hline 16 & Cansancio o sueño & $70.7 \%$ & $49.5 \%$ & $43 \%$ & $6.5 \%$ \\
\hline 17 & Mucha sed, boca seca & $64.7 \%$ & $78.6 \%$ & $21.4 \%$ & - \\
\hline 18 & $\begin{array}{l}\text { Respiraciones rápidas } \\
\text { y profundas }\end{array}$ & $16.5 \%$ & $57.1 \%$ & $23.8 \%$ & $19 \%$ \\
\hline 19 & Piel seca & $34.6 \%$ & $41.3 \%$ & $17.4 \%$ & $41.3 \%$ \\
\hline 20 & Otros & $5.3 \%$ & $66.7 \%$ & $16.7 \%$ & $16.7 \%$ \\
\hline
\end{tabular}

Tabla 2. Frecuencias de respuesta a los ítems de la escala causal.

\begin{tabular}{lll}
\hline Causas del origen & & $\begin{array}{l}\text { Frecuencia de } \\
\text { elección }\end{array}$ \\
\hline 1 & CC. Medioambientales & $5.1 \%$ \\
2 & CC. Biológicas & $71.3 \%$ \\
3 & Estilos Vida & $27.2 \%$ \\
4 & CC. Psicológicas & $22.1 \%$ \\
5 & Atención Médica & $8.8 \%$ \\
6 & CC. Sobrenaturales & $8,1 \%$ \\
7 & Otras & $1.5 \%$ \\
\hline Causas situacionales & $/ /$ & $/ /$ \\
\hline 1 & Mal día & $34.6 \%$ \\
2 & Dormir mucho/poco & $23.5 \%$ \\
3 & Saltar Tto. & $52.2 \%$ \\
4 & Saltar dieta & $81.6 \%$ \\
5 & Mucho/poco ejercicio & $70.6 \%$ \\
6 & Malos momentos & $44.1 \%$ \\
7 & Preocupaciones & $61 \%$ \\
8 & Situación Estresante & $72.8 \%$ \\
9 & Desanimado, triste, solo & $31.6 \%$ \\
10 & Otras & $0.7 \%$ \\
\hline
\end{tabular}

En la escala de control/cura, los resultados indican que casi todos los ítems presentan respuestas agrupadas en torno a dos o tres valores de la escala ( 3,4 y 5$)$, quedando los valores del otro extremo sin apenas respuestas. De ahí que se observen también desviaciones típicas por debajo de 1 y asimetría significativa en muchos de los ítems (ContPers1, ContPers2, ContPers3, ContTto1, ContTto3, ContOtro1 y Cura). 
En lo que respecta a la curtosis, la forma de la distribución que adoptan los ítems es la de la curva normal.

En el caso de los ítems sobre el curso, se encuentra que el ítem duración presenta casi todas sus respuestas en el ex- tremo superior de la escala (que va de 1 a 3). En el ítem evolución las respuestas están más repartidas, pero también existe asimetría y curtosis.

Tabla 3. Frecuencias de respuesta e índices descriptivos de los ítems de consecuencias, control/ cura y curso.

\begin{tabular}{|c|c|c|c|c|c|c|c|c|c|c|c|c|c|}
\hline Consecuencias & 1 & 2 & 3 & 4 & 5 & Mín & Máx & Media & D.T. & $M d n$ & Mo & As & $C r$ \\
\hline$\overline{\text { C.Sintomas1 (Familia) }}$ & 11 & 22 & 41 & 32 & 30 & 1 & 5 & 3.35 & 1.22 & 3 & 3 & -.235 & $-.841(*)$ \\
\hline C.Síntomas 2 (R.Sociales) & 45 & 39 & 33 & 14 & 5 & 1 & 5 & 2.22 & 1.12 & 2 & 1 & $.606(*)$ & -.454 \\
\hline C.Síntomas3 (Trabajo) & 13 & 36 & 39 & 31 & 16 & 1 & 5 & 3.00 & 1.16 & 3 & 3 & .071 & $-.848\left(^{*}\right)$ \\
\hline C.Síntomas4 (Personales) & 15 & 27 & 32 & 40 & 20 & 1 & 5 & 3.17 & 1.23 & 3 & 4 & -.211 & $-.953(*)$ \\
\hline C.Curso1 (Familia) & 17 & 27 & 40 & 34 & 18 & 1 & 5 & 3.06 & 1.21 & 3 & 3 & -.103 & $-.881(*)$ \\
\hline C.Curso2 (Rel. Sociales) & 44 & 35 & 44 & 11 & 2 & 1 & 5 & 2.20 & 1.03 & 2 & 1 & $.352(*)$ & $-.717(*)$ \\
\hline C.Curso3 (Trabajo) & 17 & 36 & 39 & 28 & 15 & 1 & 5 & 2.91 & 1.19 & 3 & 3 & .120 & $-.852(*)$ \\
\hline C.Curso4 (Personales) & 18 & 34 & 29 & 35 & 19 & 1 & 5 & 3.02 & 1.27 & 3 & 4 & -.020 & $-1.100(*)$ \\
\hline C.Control1 (Familia) & 28 & 28 & 38 & 25 & 17 & 1 & 5 & 2.81 & 1.30 & 3 & 3 & .122 & $-1.031(*)$ \\
\hline C.Control2(Rel. Sociales) & 46 & 42 & 35 & 11 & 2 & 1 & 5 & 2.12 & 1.02 & 2 & 1 & $.549(*)$ & -.459 \\
\hline C.Control3 (Trabajo) & 24 & 28 & 39 & 27 & 17 & 1 & 5 & 2.88 & 1.27 & 3 & 3 & .057 & $-.986(*)$ \\
\hline C.Control4 (Personales) & 21 & 33 & 33 & 29 & 19 & 1 & 5 & 2.94 & 1.28 & 3 & 2 & .069 & $-1.064(*)$ \\
\hline C.Futuro1 (Personales) & 1 & 9 & 32 & 50 & 43 & 1 & 5 & 3.92 & .94 & 4 & 4 & $-.554(*)$ & -.331 \\
\hline C.Futuro2 (Familia) & 4 & 16 & 34 & 45 & 37 & 1 & 5 & 3.69 & 1.08 & 4 & 4 & $-.506(*)$ & -.489 \\
\hline C.Futuro3 (Trabajo) & 12 & 28 & 38 & 31 & 27 & 1 & 5 & 3.24 & 1.23 & 3 & 3 & -.117 & $-.966(*)$ \\
\hline C.Futuro4 (Rel.Sociales) & 32 & 34 & 33 & 23 & 13 & 1 & 5 & 2.63 & 1.27 & 3 & 2 & .297 & $-.967\left(^{*}\right)$ \\
\hline Control/Cura & 1 & 2 & 3 & 4 & 5 & Mín & Máx & Media & $D . T$. & $M d n$ & Mo & As. & $C r$ \\
\hline Cont.Enf (General) & 2 & 9 & 54 & 55 & 16 & 1 & 5 & 3.54 & .84 & 4 & 4 & -.254 & .227 \\
\hline Cont.Sint (General) & 2 & 18 & 59 & 48 & 9 & 1 & 5 & 3.32 & .84 & 3 & 3 & -.145 & -.062 \\
\hline Cont.Pers1 (Personal) & & & 15 & 54 & 67 & 3 & 5 & 4.38 & .67 & 4 & 5 & $-.647(*)$ & -.666 \\
\hline Cont.Pers2 (Personal) & 2 & 8 & 28 & 61 & 37 & 1 & 5 & 3.90 & .91 & 4 & 4 & $-.741(*)$ & .418 \\
\hline Cont.Pers3 (Personal) & 6 & 11 & 47 & 64 & 6 & 1 & 5 & 3.39 & .87 & 4 & 4 & $-.867(*)$ & $.790(*)$ \\
\hline Cont.Pers4 (Personal) & 3 & 15 & 58 & 50 & 10 & 1 & 5 & 3.36 & .85 & 3 & 3 & -.271 & .165 \\
\hline Cont.Tto1 (Tratamiento) & & 1 & 23 & 65 & 47 & 2 & 5 & 4.16 & .72 & 4 & 4 & $-.374(*)$ & -.594 \\
\hline Cont.Tto2 (Tratamiento) & & 1 & 13 & 82 & 40 & 2 & 5 & 4.18 & .62 & 4 & 4 & -.334 & .330 \\
\hline Cont.Tto3 (Tratamiento) & & 1 & 12 & 70 & 52 & 2 & 5 & 4.28 & .65 & 4 & 4 & $-.526(*)$ & .079 \\
\hline Cont.Otro1 (Otros) & 1 & 8 & 30 & 51 & 46 & 1 & 5 & 3.97 & .93 & 4 & 4 & $-.627(*)$ & -.191 \\
\hline Cont.Otro2 (Otros) & 1 & 14 & 43 & 51 & 26 & 1 & 5 & 3.64 & .93 & 4 & 4 & -.234 & -.534 \\
\hline Cura & 71 & 30 & 19 & 11 & 5 & 1 & 5 & 1.88 & 1.14 & 1 & 1 & $1.148(*)$ & .340 \\
\hline$\overline{C u r s o}$ & 1 & 2 & 3 & 4 & $1 /$ & Mín & Máx & Media & $D . T$. & $M d n$ & Mo & $A s$ & $C r$ \\
\hline Duración & 1 & 9 & 126 & $1 /$ & // & 1 & 3 & 2.91 & .29 & 3 & 3 & $-3.914(*)$ & $16.172\left(^{*}\right)$ \\
\hline Evolución & 26 & 10 & 60 & 40 & $1 /$ & 1 & 4 & 2.83 & 1.05 & 3 & 3 & $-.667(*)$ & $-.731\left(^{*}\right)$ \\
\hline
\end{tabular}

En lo se refiere a la homogeneidad de los ítems, se observa en la Tabla 4 cómo las correlaciones ítem-total corregidas son adecuadas para la mayoría de los ítems (por encima de .30), excepto el algunos ítems de la escala de control/cura que se separan ligeramente del criterio, aunque no de forma significativa ( $p>.05$ en todos los casos).

En cuanto a la capacidad de discriminación del ítem, teniendo en cuenta los grupos extremos de puntuaciones totales en la escala (superando y por debajo de los percentiles 73 y 27 , respectivamente), se observa que absolutamente todos los ítems tienen la capacidad de discriminar entre los grupos.

\section{Análisis de Factorial Exploratorio $(A F E)$ para la es- cala de consecuencias y la de control/cura}

Como puede apreciarse en la Tabla 5, los resultados indican que los ítems se agrupan en tantos factores como sub- componentes fueron especificados en su respectiva definición.

En la escala de consecuencias la prueba de adecuación muestral Kaiser-Mayer-Olkin es .85 y el índice de esfericidad de Barlett es significativo $(\phi<.00)$. El análisis con rotación Oblimin ofrece una salida con 4 factores que explicarían el $76.01 \%$ de la varianza. El Factor I agrupa ítems de consecuencias familiares, el Factor II agrupa conjuntamente, sin distinguir entre ellas, las consecuencias personales y laborales. El Factor III agrupa a las consecuencias en las relaciones sociales, y finalmente, el Factor IV agrupa a los ítems relativos a consecuencias de todo tipo, pero con la particularidad de ser consecuencias futuras. El ítem "conscon3", de consecuencias laborales, satura más en este factor que en ninguno, pero siendo sus saturaciones similares también en el Factor II, se ha incluido en este último por su proximidad semántica con el resto de los ítems. 
Tabla 4. Otros descriptivos y correlación Item-Total Corregida.

\begin{tabular}{|c|c|c|c|}
\hline Consecuencias & $\begin{array}{c}\text { Correlación } \\
\text { Item-Total Corregida }\end{array}$ & MP27 & MP73 \\
\hline C. Síntomas1 & .516 & 2.54 & $4.05^{*}$ \\
\hline C. Síntomas2 & 619 & 1.45 & $3.02 *$ \\
\hline C. Síntomas3 & .634 & 2.02 & $3.94 *$ \\
\hline C. Síntomas4 & 656 & 2.02 & $4.00^{*}$ \\
\hline C. Curso1 & .628 & 2.14 & $3.86^{*}$ \\
\hline C. Curso2 & .663 & 1.40 & $3.00 *$ \\
\hline C. Curso3 & 682 & 1.85 & $3.91 *$ \\
\hline C. Curso4 & 651 & 1.88 & $3.91 *$ \\
\hline C. Control1 & .621 & 1.88 & $3.75^{*}$ \\
\hline C. Control2 & .683 & 1.42 & $3.02 *$ \\
\hline C. Control3 & .736 & 1.74 & $4.19 *$ \\
\hline C. Control4 & .678 & 1.88 & $3.97 *$ \\
\hline C. Futuro1 & .579 & 2.94 & $4.69 *$ \\
\hline C. Futuro2 & .554 & 2.62 & $4.47 *$ \\
\hline C. Futuro3 & .502 & 2.11 & $4.33^{*}$ \\
\hline C. Futuro4 & .562 & 1.51 & $3.77 *$ \\
\hline \multicolumn{4}{|l|}{$* p<.05$} \\
\hline Control/Cura & $\begin{array}{c}\text { Correlación } \\
\text { Item-Total Corregida }\end{array}$ & MP27 & MP73 \\
\hline Cont.Enf & .384 & 2.97 & $4.02 *$ \\
\hline Cont.Sint & .317 & 2.75 & $3.61 *$ \\
\hline Cont.Pers1 & .395 & 4.00 & $4.80^{*}$ \\
\hline Cont.Pers2 & .473 & 3.25 & $4.41 *$ \\
\hline Cont.Pers3 & .365 & 2.91 & $3.77 *$ \\
\hline Cont.Pers4 & .342 & 2.83 & $3.72 *$ \\
\hline Cont.Tto1 & .295 & 3.86 & $4.41 *$ \\
\hline Cont.Tto2 & .283 & 3.97 & $4.44 *$ \\
\hline Cont.Tto3 & .273 & 4.05 & $4.55^{*}$ \\
\hline Cont.Otro1 & .286 & 3.41 & $4.41 *$ \\
\hline Cont.Otro2 & .345 & 3.11 & $4.25^{*}$ \\
\hline Cura & .211 & 1.13 & $3.05^{*}$ \\
\hline Curso & & MP27 & MP73 \\
\hline Duración & - & 2.76 & $3.00^{*}$ \\
\hline Evolución & - & 1.41 & $4.00^{*}$ \\
\hline
\end{tabular}

Para la escala de control/cura, los índices iniciales son de .63 para la adecuación muestral y la prueba de esfericidad significativa también, como en el caso anterior. En este caso, el análisis factorial resulta en un modelo formado por 3 factores, que explicarían un $51.58 \%$ de la varianza. El Factor I agrupa los ítems de control general, personal y la cura, el Factor II los del control de otras personas y el Factor III incluye los ítems del control a través del tratamiento.

Para el análisis de la fiabilidad se realizó el test-retest con tres días de margen entre una aplicación y otra. Sólo una parte de la muestra total $(n=63)$ accedió a volver a cumplimentar los instrumentos. En los análisis se incluyó: la correlación entre el test y el re-test de las puntuaciones totales en las escalas cuantitativas, discrepancias en la elección en las escalas cualitativas, la correlación intrasujeto considerando todos los ítems de la escala y la consistencia interna ( $\alpha$ de Cronbach).
Tabla 5. Resultados del Análisis de Factorial Exploratorio con rotación Oblimin.

\begin{tabular}{|c|c|c|c|c|c|c|c|}
\hline \multirow{2}{*}{\multicolumn{4}{|c|}{ CONSECUENCIAS }} & \multicolumn{4}{|c|}{ Factor } \\
\hline & & & & I & II & III & IV \\
\hline \multirow{13}{*}{ Futuras } & & & conssit & 0.841 & & & \\
\hline & & & conscur1 & 0.832 & & & \\
\hline & Fat & & conscon 1 & 0.643 & & & \\
\hline & Fa1 & & consfu2 & 0.680 & & & 0.583 \\
\hline & $\operatorname{Tr}$ & & conssi3 & \multicolumn{3}{|c|}{0.693} & \\
\hline & $\operatorname{Tr}$ & & conscur3 & \multicolumn{3}{|c|}{0.733} & \\
\hline & $\operatorname{Tr}$ & & $\operatorname{conscon} 3$ & \multicolumn{3}{|c|}{0.629} & 0.663 \\
\hline & Per & nales & conscon4 & \multicolumn{3}{|c|}{0.759} & \\
\hline & Pet & nales & conssi4 & \multicolumn{3}{|c|}{0.798} & \\
\hline & & nales & conscurt & \multicolumn{3}{|c|}{0.819} & \\
\hline & Re & iones & conssi2 & \multicolumn{4}{|c|}{0.768} \\
\hline & Re & iones & conscur2 & \multicolumn{4}{|c|}{0.872} \\
\hline & Re & iones & $\operatorname{conscon} 2$ & \multicolumn{4}{|c|}{0.944} \\
\hline Futuras & Pet & nales & consfu1 & & & & 0.695 \\
\hline Futuras & $\operatorname{Tr}$ & & consfu3 & & & & 0.868 \\
\hline \multirow[t]{2}{*}{ Futuras } & \multicolumn{3}{|c|}{ Relaciones } & & & & 0.623 \\
\hline & & & & \multicolumn{4}{|c|}{ Factor } \\
\hline \multicolumn{4}{|c|}{ CONTROL/CURA } & $\mathbf{I}$ & & & III \\
\hline \multicolumn{2}{|c|}{ General } & \multicolumn{2}{|c|}{ contrenf } & 0.604 & & & \\
\hline \multicolumn{2}{|c|}{ General } & \multicolumn{2}{|c|}{ controsi } & 0.567 & & & \\
\hline \multicolumn{2}{|c|}{ Personal } & \multicolumn{2}{|c|}{ contper1 } & 0.508 & & & \\
\hline \multicolumn{2}{|c|}{ Personal } & \multicolumn{2}{|c|}{ contper2 } & 0.549 & & & \\
\hline \multicolumn{2}{|c|}{ Personal } & \multicolumn{2}{|c|}{ contper3 } & 0.532 & & & \\
\hline \multicolumn{2}{|c|}{ Personal } & \multicolumn{2}{|c|}{ contpert } & 0.457 & & & \\
\hline \multicolumn{2}{|c|}{ Tratamiento } & contt & & & & 73 & \\
\hline Tratami & & contt & & & & 18 & \\
\hline Tratami & ato & contt & & & & 94 & \\
\hline Otros & & conot & & & & & 0.653 \\
\hline Otros & & conot & & & & & 0.823 \\
\hline Cura & & cura & & 0.371 & & & \\
\hline
\end{tabular}

\section{Estabilidad de las puntuaciones: test-retest y dis-} crepancias

En la escala de identidad se obtuvieron indicadores de la estabilidad significativos tanto en el numero total de sintomas como en las puntuaciones sobre la relación sintoma-enfermedad $\left(r_{\mathrm{t}-\mathrm{r}}=.75\right.$ y $r_{\mathrm{t}-\mathrm{r}}=.80$, respectivamente $)$. Dicho esto, se podría cuestionar que el primero sea un dato suficiente por no tratarse de la estabilidad específica de la escala en relación a los síntomas concretos, sino al número. Por eso, en segundo lugar, se realizó también el test-retest de los síntomas concretos, analizando las discrepancias en la elección de los mismos. De la misma manera se procedió a analizar la estabilidad de las causas que componen la sub-escala Causal (discrepancias en la elección de las causas entre las dos aplicaciones de la prueba). En la Tabla 6 aparecen los resultados.

En la mayoría de los síntomas de la escala de identidad no existen discrepancias resaltables. Como se observa en la Tabla 6, la discrepancia promedio de la escala es del 13.2\%. En lo que a las causas se refiere ocurre exactamente igual; en términos generales podemos decir que estas respuestas muestran también estabilidad a lo largo del tiempo, ya que en promedio esta discrepancia no llega a alcanzar el 15\%. 
Tabla 6. Discrepancias en los síntomas de la escala de identidad y en las causas de la escala causal

\begin{tabular}{|c|c|c|c|c|c|}
\hline Síntomas & $\begin{array}{c}\text { Respuestas } \\
\text { discrepantes }\end{array}$ & $\%$ & $\begin{array}{c}\text { Causas } \\
\text { Generales }\end{array}$ & $\begin{array}{c}\text { Respuestas } \\
\text { discrepantes }\end{array}$ & $\%$ \\
\hline 1 & 6 & 11.3 & 1 & 4 & 7 \\
\hline 2 & 8 & 15.1 & 2 & 5 & 8.8 \\
\hline 3 & 12 & 22.6 & 3 & 11 & 19.3 \\
\hline 4 & 9 & 17 & 4 & 6 & 10.5 \\
\hline 5 & 17 & 32.1 & 5 & 3 & 5.3 \\
\hline 6 & 9 & 17 & 6 & 2 & 3.5 \\
\hline 7 & 7 & 13.2 & 7 & 1 & 1.8 \\
\hline 8 & 8 & 15.1 & $\begin{array}{c}\text { Situaciones } \\
\text { antecedentes }\end{array}$ & $\begin{array}{c}\text { Respuestas } \\
\text { discrepantes }\end{array}$ & $\%$ \\
\hline 9 & 7 & 13.2 & 1 & 11 & 19.3 \\
\hline 10 & 3 & 5.7 & 2 & 12 & 21.1 \\
\hline 11 & 8 & 15.1 & 3 & 6 & 10.5 \\
\hline 12 & 9 & 17 & 4 & 10 & 17.5 \\
\hline 13 & 8 & 15.1 & 5 & 13 & 22.8 \\
\hline 14 & 6 & 11.3 & 6 & 15 & 26.3 \\
\hline 15 & 7 & 13.2 & 7 & 18 & 31.6 \\
\hline 16 & 13 & 24.5 & 8 & 13 & 22.8 \\
\hline 17 & 10 & 18.9 & 9 & 8 & 14 \\
\hline 18 & 12 & 22.6 & 10 & 3 & 5.4 \\
\hline 19 & 9 & 17 & \multirow{2}{*}{\multicolumn{3}{|c|}{$\begin{array}{l}\text { Discrepancia media en la escala } \\
=14.55 \%\end{array}$}} \\
\hline 20 & 5 & 9.4 & & & \\
\hline
\end{tabular}

Test-Retest para las escalas cuantitativas (consecuencias, control-cura y curso)

Las correlaciones test-retest que se han obtenido para las escalas de consecuencias, control/cura y curso indican que las puntuaciones se mantienen moderadamente estables a lo largo del tiempo $\left(r_{\mathrm{t}-\mathrm{r}}=.63, r_{\mathrm{t}-\mathrm{r}}=.68, r_{\mathrm{t}-\mathrm{r}}=.81\right.$, respectivamente $)$. Todas las correlaciones son significativas $(p<.01)$.

\section{Test-Restest intrasujeto}

Observando la Tabla 7, se puede comprobar cómo las correlaciones se encuentran en un rango que va desde .70 a .99 . Se podría decir que, en general, los datos indican que las respuestas individualmente consideradas se mantienen estables en el tiempo.

\section{Consistencia interna: sub-escalas de consecuencias y control-cura}

Finalmente, los resultados sobre la consistencia interna indicaron un $\alpha=.91 \mathrm{y} \alpha=.68$, respectivamente. Ambas escalas tienen un número diferente de ítems (16 y 12 , respectivamente), lo que hace que los índices no sean comparables. Sin embargo, se podría decir que la escala de consecuencias presenta mayor consistencia interna que la de control/cura, que ha resultado por debajo de .70 . Recuérdese que algunos de sus ítems mostraban una correlación ítem-total (corregida) menor de .30 .
Tabla 7. Correlaciones intrasujeto entre el Test y el Retest para todos los ítems

\begin{tabular}{|c|c|c|c|}
\hline Sujetos & $\boldsymbol{R}_{\mathrm{t}-\mathrm{r}}$ TODA ESCALA & Sujetos & $\boldsymbol{R}_{\mathrm{t}-\mathrm{r}}$ TODA ESCALA \\
\hline 1 & .92 & 28 & .93 \\
\hline 2 & .95 & 29 & .93 \\
\hline 3 & .93 & 30 & .93 \\
\hline 4 & .87 & 31 & .96 \\
\hline 5 & .73 & 32 & .99 \\
\hline 6 & .90 & 33 & .97 \\
\hline 7 & .92 & 34 & .91 \\
\hline 8 & .90 & 35 & .74 \\
\hline 9 & .81 & 36 & .91 \\
\hline 10 & .93 & 37 & .88 \\
\hline 11 & .95 & 38 & .89 \\
\hline 12 & .87 & 39 & .94 \\
\hline 13 & .90 & 40 & .89 \\
\hline 14 & .92 & 41 & .87 \\
\hline 15 & .87 & 42 & .92 \\
\hline 16 & .95 & 43 & .94 \\
\hline 17 & .87 & 44 & .90 \\
\hline 18 & .88 & 45 & .88 \\
\hline 19 & .94 & 46 & .89 \\
\hline 20 & .80 & 47 & .94 \\
\hline 21 & .90 & 48 & .85 \\
\hline 22 & .84 & 49 & .92 \\
\hline 23 & .90 & 50 & .97 \\
\hline 24 & .74 & 51 & .91 \\
\hline 25 & .92 & 52 & .96 \\
\hline 26 & .92 & 53 & .88 \\
\hline 27 & .91 & 54 & .88 \\
\hline
\end{tabular}

\section{Conclusiones}

Como ya se ha podido comprobar se han hecho distintos análisis para obtener información sobre el comportamiento estadístico de los elementos que fueron elaborados tras las especificaciones del test y de los ítems. Así, en lo que se refiere a las escalas de identidad y causas, la información obtenida indica la adecuación de los ítems desarrollados. Parece que los indicadores que se han elegido para representar las definiciones son acordes a éstas. Aunque se ha observado cómo hay síntomas más o menos frecuentes, así como atribuciones causales más o menos comunes esto es, en principio, esperable si consideramos la enfermedad de que se trata. Sin embargo, realizar un análisis de los ítems considerando cada causa/síntoma individualmente y obtener resultados en relación a otras variables puede ser de gran interés para obtener más información sobre su comportamiento y también sobre la validez de las definiciones, tal y como se ha apuntado (De los Santos-Roig, Pérez, Ruiz-González, GuardiaArchilla y Martínez-García, 2010; Ruggiero, Goodie y Morris, 1999). Quizás eso deba ser tenido en cuenta en futuros trabajos.

Los ítems de la escala de consecuencias son los que mejores resultados han mostrado. De hecho, se puede concluir que casi todos los de esta escala presentan unos índices descriptivos adecuados: variabilidad en la frecuencia de respuestas a cada uno de los valores de la escala tipo Likert a la 
que van asociados, medias cercanas al punto medio, desviación típica por encima de 1, relación con los ítems de su escala, distinción entre grupos de puntuaciones extremas, etc.

Los resultados de los descriptivos para los ítems de control/cura y curso han podido resultar más llamativos (por su variabilidad más baja, fundamentalmente). La mayoría de los ítems no presentan elecciones en todas sus posibles respuestas. La media, mediana y moda, indican que las respuestas de casi todos los ítems se alejan del punto central de la escala, así como su desviación típica es pequeña, ya que todas las respuestas se encuentran distribuidas entre tres valores (3, 4 y 5 generalmente). La explicación sobre esto podría estar en que la mayoría de las personas diabéticas de la muestra, cuando responden a estos ítems, parten de la base de cierto control percibido sobre la enfermedad (del tipo que sea y referida al aspecto que sea) ya que sus respuestas casi siempre están por encima de 3 puntos. Son muy pocos los participantes que contestan que perciben algo o ningún control. Estas personas que asisten a programas de Educación Diabetológica conocen perfectamente la importancia que las estrategias de auto-cuidado así como la auto-administración del tratamiento tienen en el control de su enfermedad. Otra cosa es cómo, y durante cuánto tiempo lo lleven a cabo (Knight, Dornan y Bundy, 2006). De todas formas, aunque la explicación pudiera estar en las características de la muestra, no hay que perder de vista que trabajamos con unos ítems iniciales y que se trata de los primeros análisis exploratorios sobre su comportamiento. Además, no hay que olvidar que en alguno de los ítems las correlaciones con el total de la escala han resultado por debajo de .30, aunque no significativamente. Esto puede estar indicando que los subcomponentes del control son independientes entre sí, e incluso que sería necesario considerar la pertinencia de incluir un mayor número de ítems.

Por su parte el análisis factorial exploratorio ha permitido corroborar la estructura de la definición, tanto de las consecuencias, como del control. Exceptuando un ítem de la escala de consecuencias ("conscon3") que ha saturado ligeramente más en otro factor que en el suyo propio, lo más resaltable es la aparición de un factor que agrupa los ítems de consecuencias laborales y personales. Analizado con detenimiento el contenido de los ítems, no tenemos una explicación más allá de la pura especulación para dar respuesta a este resultado. Por su parte, ocurre algo similar en la escala de control/cura. En este caso, aparece un factor (Factor I) que agrupa a los ítems de control general, personal y cura lo que nos lleva a reflexionar sobre qué puede estar primando a la hora de responder a ambos (el contenido semántico, el orden de los ítems en la escala, etc.). Como el $A F E$ puede cambiar al variar la muestra $\mathrm{y}$, considerando que se ha tratado sólo de una exploración inicial para comprobar la definición, no iremos más allá en la interpretación de los resultados. Se dejará así a expen- sas de contar con un tamaño muestral mayor para poder realizar un análisis factorial confirmatorio, lo que sin duda ayudará a interpretar el porqué de esos resultados y, también, a corroborar lo propuesto en las definiciones. Al hilo de esto no se quiere acabar sin decir que sería necesario utilizar otro tipo de muestras para comprobar si lo que se pretende sondear está suficientemente representado en los ítems (Landsheer y Boeije, 2010) y, sobre todo, cómo son comprendidos y/o procesados éstos por el que contesta la prueba, como han planteado algunos autores (French, Cooke, McLean, Williams y Sutton, 2007; Van Oort, Schröder y French, 2011). En definitiva, no hay que perder de vista el contenido del item y los procesos que se ven implicados al recoger información mediante el auto-informe. Lo que está claro es que el uso de metodologías aplicadas a la elaboración de instrumentos, como las entrevistas cognitivas o los métodos de pretest cognitivo (Castillo-Díaz, Padilla-García, GómezBenito y Andrés-Valle, 2010; Drennan, 2003; Willis, 2004), podrían ser de muchísima utilidad para mejorar el proceso de construcción de ítems así como el análisis de éstos.

Por último, la fiabilidad ha sido estimada a través de diversas estrategias. Por un lado, los análisis sobre la estabilidad a través del test-retest, han resultado ser adecuados para todas las escalas analizadas (identidad (número de sintomas), consecuencias, control/ cura y curso). De hecho, los resultados fueron similares a los obtenidos con otros instrumentos que miden la RCE, como el IPQ-Illness Perception Questionnaire- en su versión original, en la revisada y en la breve (Broadbent, Petrie, Main y Weinman, 2006; Moss-Morris et al., 2002; Weinman, Petrie, Moss-Morris y Horne, 1996), así como en su versión española (Beléndez-Vázquez et al., 2005). Igual ocurre con la consistencia interna (a través del alfa de Cronbach), que ha sido estudiada en las escalas de consecuencias y control/ cura, obteniéndose valores adecuados y comparables a los de los trabajos citados anteriormente. Finalmente se ha analizado la fiabilidad de toda la escala (considerando las cinco sub-escalas conjuntamente), teniendo en cuenta todos sus ítems, para cada participante individualmente (test-retest intrasujeto). Se puede concluir que, en general, se encuentran datos que indican la estabilidad de las puntuaciones a lo largo del tiempo.

En definitiva, parece que el proceso de construcción de ítems a partir del establecimiento de la definición operativa del constructo Representación de la Enfermedad (De los SantosRoig y Pérez, 2013) ha resultado adecuado, y se puede considerar que la primera versión de la ERCE cumple con los criterios establecidos en cuanto al comportamiento de los ítems y a la fiabilidad de las puntuaciones. Quedarían por discutirse las evidencias obtenidas sobre la validez de esas puntuaciones, pero éstas serán objeto de análisis y comentario en futuros trabajos (mientras tanto, los interesados pueden consultar las conclusiones en este sentido en De los Santos-Roig, 2009). 


\section{Referencias}

AERA, APA y NCME, (1999). Standards for educational and psychological tests. Washington DC: American Psychological Association, American Educational Research Association, National Council on Measurement in Education.

Beléndez-Vázquez, M., Bermejo-Alegría, R. M. y García-Ayala, M. D. (2005). Estructura factorial de la versión española del Revised Illness Perception Questionnaire en una muestra de hipertensos. Psicothema, 17, 318-324.

Broadbent, E., Donkin, L. y Stroh J. C. (2011). Illness and Treatment Perceptions Are Associated With Adherence to Medications, Diet, and Exercise in Diabetic Patients. Diabetes Care, 34, 338-340.

Broadbent, E., Petrie, K. J., Main, J. y Weinman, J. (2006). The Brief Illness Perception Questionnaire. Journal of Psychosomatic Research, 60, 631- 637.

Castillo-Díaz, M., Padilla-García, J. L., Gómez-Benito, J. y Andrés-Valle, A. (2010). A productivity map of cognitive pretest methods for improving survey questions. Psicothema, 22, 475-481.

De los Santos-Roig, M. (2009). Evaluación de la Representación de la Enfermedad: Creación de la Escala para la Representación Cognitiva de la Enfermedad, ERCE (Tesis doctoral no publicada). Universidad de Granada, Granada.

De los Santos-Roig, M. y Pérez-Meléndez, C. (2013). De la definición semántica a los ítems a través de las tablas de especificaciones: Elaboración de la Escala sobre Representación de la Enfermedad, ERCE Anales de Psicologia, 29(2), 345-359.

De los Santos-Roig, M., Pérez, C., Ruiz-González, I., Guardia-Archilla, T. y Martínez-García, A. (2010). Atribuciones Causales en la enfermedad: La importancia de las atribuciones especificas en la relación con otros componentes de la Representación de la Enfermedad, el Afrontamiento y el Ajuste del paciente. Póster presentado en el VII Congreso Iberoamericano de Psicología. Oviedo, España.

Drennan, J. (2003) Cognitive interviewing: verbal data in the design and pretesting of cuestionnaires. Journal of advance nursing, 42(1), 57-69.

French, D. P., Cooke, R., McLean, N., Williams, M. y Sutton, S. (2007). What do people think about when they answer theory of planned behaviour questionnaires? A "think aloud" study. Journal of Health Psychology, 12, 672-687.

Hagger, M. y Orbell, S. (2003). A meta-analytic review of the common-sense model of illness representations. Psychology and Health, 18, 141-184.

Hoving, J. L., Van der Meer, M., Volkova, A. Y. y Frings-Dresen, M. H. W. (2010). Illness perceptions and work participation: a systematic review. International Archives of Occupational and Environmental Health, 83, 595-605.
Knight K. M., Dornan T., Bundy C. (2006) The diabetes educator: trying hard, but must concentrate more on behaviour. Diabetes Medicine, 23(5), 485-501.

Landsheer, J. A. y Boeije, H. R. (2010). In search of content validity: facet analysis as a qualitative method to improve questionnaire design. An application in health research. Ouality and Ouantity, 44, 59-69.

Leventhal, H., Benyamini, Y., Brownlee, S., Diefenbach, M., Leventhal, E. A., Patrik-Miller, L. y Robitaille, C. (1997). Illness Representations: Theoretical Foundations. En K. J. Petrie y J. A. Weinman, (Eds.), Perceptions of Health and Illness (pp. 19-46). Amsterdam: Harwood Academic Publishers.

Leventhal, H., Brissette, I. y Leventhal, E. A. (2003). The Common-Sense Model of self-regulation of health and illness. En L.D. Cameron y H. Leventhal, (Eds.), The self-regulation of health and illness behaviour (pp. 4265). London y New York: Routledge.

McSharry, J., Moss-Morris, R. y Kendrick, T. (2011). Illness perceptions and glycaemic control in diabetes: a systematic review with meta-analysis. Diabetic Medicine, 28, 1300-1310.

Moss-Morris, R., Weinman, J., Petrie, K. J., Horne, R., Cameron, L. D. y Buick, D. (2002). The revised illness perception questionnaire (IPQ-R). Psychology and Health, 17, 1-16.

Ruggiero, K. J., Goodie, J. L. y T. L. Morris, (1999). Using item analysis to facilitate interpretation of empirical findings. Journal of Behavior Therapy and Experimental Psychiatry, 30, 63-69.

Van Oort, L., Schröder, C. y French, D. P. (2011). What do people think about when they answer the Brief Illness Perception Questionnaire? A "think aloud" study. British Journal of Health Psychology, 16(2), 231-245.

Van-der Hofstadt, C. J. y Rodríguez-Marín, J. (1997). Adaptación de un cuestionario para la medida de la representación de la enfermedad. Psicothema, 9, 237-245.

Weinman, J., Petrie, K. J. Moss-Morris, R. y Horne, R. (1996). The illness perception questionnaire: a new method for assessing the cognitive presentations of illness. Psychology and Health, 11, 431-440.

Willis, G. B. (2004). Cognitive Interview Revisiting: A useful technique, in Theory? En S. Presser, J. Rothgeb, M. P. Couper, J. T. Lessler, E. Martin, J. Martin y E.Singer (Eds.), Methods for Testing and Evaluating Survey Questions (pp. 23-43). Nueva Jersey: John Wiley and Sons.

(Artículo recibido: 5-12-2012; revisado: 21-2-2013; aceptado: 21-6-2013) 
Anexo. Versión de la ERCE tras el análisis de ítems

\section{ESCALA IDENTIDAD}

A continuación aparece una lista con una serie de síntomas. Queremos que nos indiques cuáles de ellos ESTÁN PRESENTES EN TU VIDA, es decir padeces FRECUENTEMENTE, AUNQUE NO SEA DIARIAMENTE. Para ello, marca con una cruz la casilla que aparece junto a cada uno. Posteriormente, sobre cada síntoma marcado, queremos que nos indiques si crees que está relacionado o no con TU DIABETES. Si tienes alguna duda, levanta la mano y te atenderé de inmediato.

\begin{tabular}{|l|lcc|}
\hline & MARCA CON UNA CRUZ & ¿Crees que los síntomas que has marcado están relacionados con TU Diabetes? \\
\hline Sudoración & SI & NO & A VECES \\
\hline Temblores & SI & NO & A VECES \\
Nerviosismo & SI & NO & A VECES \\
\hline Hambre & SI & NO & A VECES \\
\hline Debilidad & SI & NO & A VECES \\
\hline Palpitaciones & SI & NO & A VECES \\
Ardores & SI & NO & A VECES \\
\hline Hormigueo (en la boca, en los hombros, etc.) & SI & NO & A VECES \\
Nauseas & SI & NO & A VECES \\
Dolor de cabeza & SI & NO & A VECES \\
Dificultad para hablar o moverse & SI & NO & A VECES \\
Agresividad & SI & NO & A VECES \\
\hline Problemas para ver bien & SI & NO & A VECES \\
Desorientación & SI & NO & A VECES \\
Mareo & SI & NO & A VECES \\
Cansancio o sueño & SI & NO & A VECES \\
Mucha sed, boca seca & SI & NO & A VECES \\
Respiraciones rápidas y profundas & SI & NO & A VECES \\
Piel seca & SI & NO & A VECES \\
Otras & SI & NO & A VECES \\
\hline
\end{tabular}

\section{ESCALA CAUSAL}

Como sabes, las enfermedades pueden estar causadas por diversos factores o situaciones. Ahora queremos que nos indiques, de todas las causas que se enumeran a continuación, aquéllas que tú crees que han favorecido que tu enfermedad se desarrollara en un principio, es decir, que tú consideres que fueron las que participaron en el origen de tu enfermedad. Después, queremos que pienses en los síntomas y en cuáles de las causas que aparecen pueden, a tu juicio, desencadenar o estar presentes en la aparición de los síntomas que habitualmente padeces. Para ello, junto a cada causa aparece una casilla. Marca con una cruz la causa que creas está relacionada con el origen de la enfermedad o con la aparición de sintomatología. Por supuesto, si no crees que esa causa tenga nada que ver ni con la enfermedad ni con los síntomas, déjala sin tachar. Si tienes alguna duda, levanta la mano y te atenderé de inmediato.

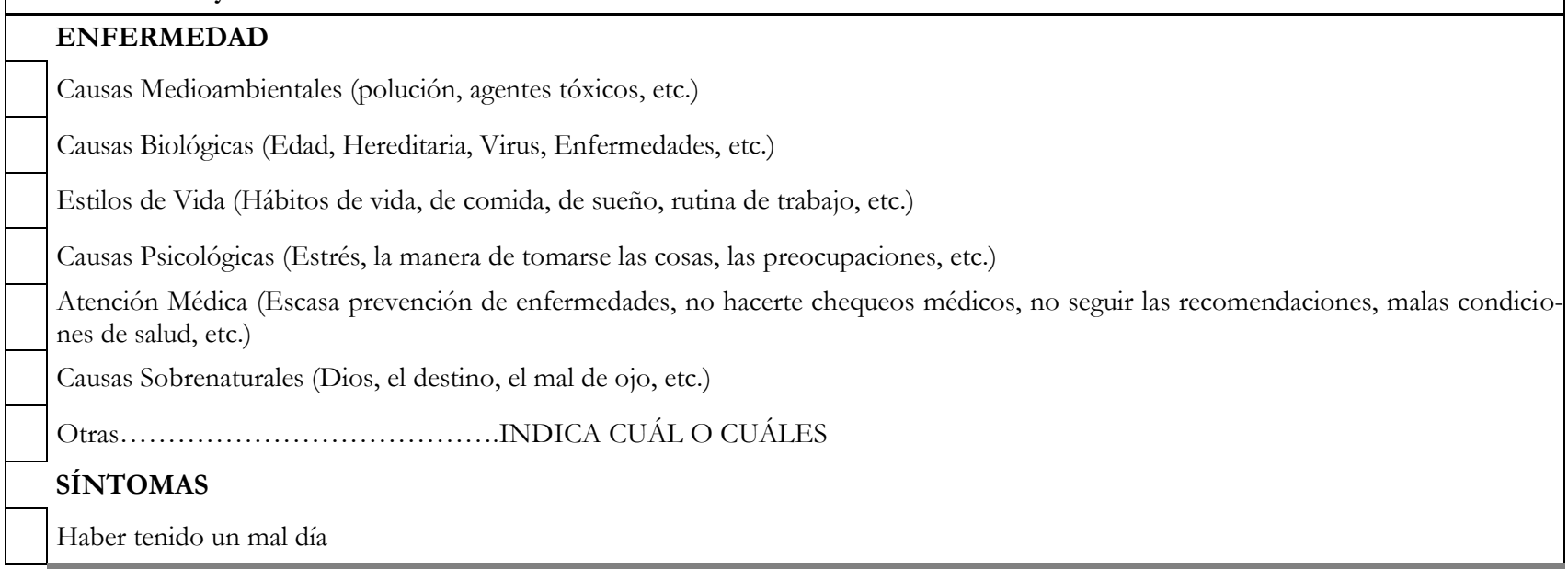




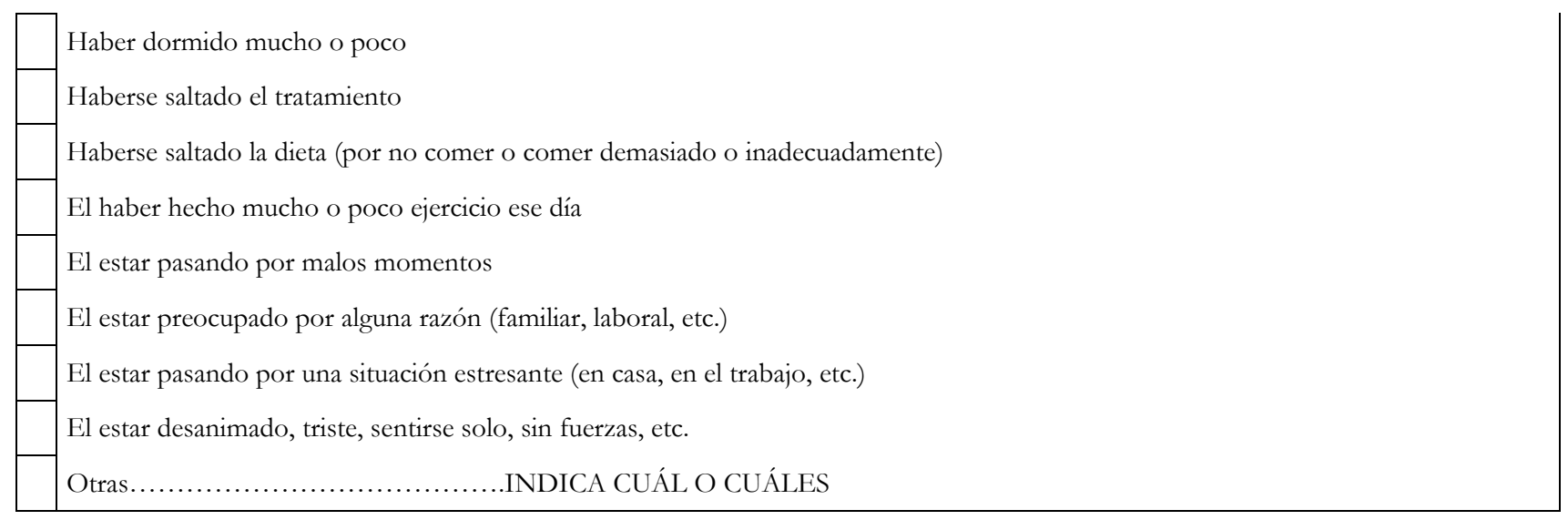

\section{ESCALA CONSECUENCIAS}

Ahora, nos interesa saber cuáles son las consecuencias que la Diabetes tiene sobre ti y para ello vamos a centrarnos en este momento SOLO en un aspecto de ésta. Concretamente queremos que pienses en TUS SÍNTOMAS, y en qué consecuencias tienen éstos sobre ti o sobre tu vida Cuando hablamos de síntomas nos referimos a aquellos propios de la enfermedad que aparecen en tu día a día. Sabemos que hay momentos del día en los que te encuentras mal (temblores, mareo, sudoración, etc) y, por ejemplo, te despiertas en medio de la noche, o momentos en los que debes dejar de hacer lo que estabas haciendo hasta recuperarte.

Sabemos que diariamente puedes padecer cualquiera de estos síntomas y queremos saber si esas sensaciones te repercuten de alguna manera en tu vida y en tu entorno. Sobretodo queremos saber en qué grado te afectan. Para ello se te van a presentar una serie de preguntas que deberás responder, rodeando con un círculo un número entre el 1 y el 5 , de manera que 5 significaría que los síntomas te afectan mucho y 1 que no te afectan nada

Siguiendo estas instrucciones, lee las siguientes preguntas y marca tu respuesta. Por favor, no dejes ninguna pregunta sin contestar.

- De 1 a 5, ¿cómo afectan los sintomas de tu diabetes a tu propia familia? (C.Sintomas1)

- ¿Y en tus relaciones sociales? (C.Sintomas2)

- Piensa en tu trabajo u obligaciones diarias sean éstas como sean, es decir, remuneradas o no, domésticas, laborales, etc. ¿Cómo te afectan los sintomas de la enfermedad? (C.Sintomas3)

- Indica ahora, ¿cómo te afectan personalmente los sintomas de esta enfermedad, es decir, a ti mismo como persona; en tu estado de ánimo, en tu manera de ver la vida, en tus proyectos, etc.? (C.Sintomas4)

ESCALA DE RESPUESTA: No afectan nada (1) - Afectan mucho (5)

Siguiendo con la misma lógica que en el caso anterior, nos gustaría ahora que pensaras sólo en cómo tu enfermedad, como tal, se presenta diariamente en tu vida desde que fue diagnosticada. Es decir, nos referimos a cómo la enfermedad "cambia" o se va comportando durante el día, por ejemplo, con la aparición de los síntomas en determinados momentos (por la noche, por la mañana, etc.).

Bien, todo ello, al igual que ocurría anteriormente con los síntomas, puede afectarte de alguna manera y en concreto, tanto a ti personalmente como a diversos aspectos de tu vida. Por eso, ahora vamos a preguntarte por el grado en el que ese comportamiento diario de la enfermedad te afecta a ti y a cuanto te rodea. Recuerda que para contestar deberás rodear un número del 1 al 5, donde el 5 sería indicativo de que te afecte mucho y el 1 de que no te afecte nada.

- ¿En qué grado afecta la evolución diaria de tu enfermedad a tu familia? (C.Curso1)

- ¿Y en tus relaciones sociales? (C.Curso2)

- Piensa en su trabajo u obligaciones diarias sean éstas como sean, es decir, remuneradas o no, domésticas, laborales, etc. ¿Cómo te afecta la evolución diaria de la enfermedad (C.Curso3)

- Indica ahora, ¿cómo te afecta personalmente, es decir, a ti mismo como persona; en tu estado de ánimo, en tu manera de ver la vida, en tus proyectos, etc.? (C.Curso4)

ESCALA DE RESPUESTA: No afecta nada (1) - Afecta mucho (5)

Ahora nos vamos a centrar en las estrategias que pones en marcha para manejar tu enfermedad Es decir, nos centraremos en todo aquello que haces para mantenerla controlada, como por ejemplo, los análisis continuos de sangre, la dieta restrictiva, pincharte la insulina antes de cada comida, comer a determinadas horas, rutinas de vida que no alteren tu ritmo cotidiano. Estas conductas o estrategias que debes realizar, pueden afectarte a ti o a algunos aspectos de tu vida en general.

Indica, como en las preguntas anteriores, el grado en el que te afectan todas estas situaciones anteriormente descritas. Para ello usa como siempre, los números del 1 al 5 que aparecen junto a cada pregunta.

- ¿En qué grado afecta a tu familia todo lo que tienes que hacer para controlar tu enfermedad? (C.Control1) 
- ¿Y en tus relaciones sociales? (C.Control2)

- Piensa en tu trabajo, ¿en qué grado te afecta todo lo que necesitas hacer por tu enfermedad? (C.Control3)

- Indica ahora ¿cómo te afecta todo esto personalmente, es decir, a ti mismo como persona; en tu estado de ánimo, en tu manera de ver la vida, en tus proyectos, etc.? (C.Control4)

ESCALA DE RESPUESTA: No afecta nada (1) - Afecta mucho (5)

Como sabes, hasta el día de hoy tu enfermedad puede ser considerada como crónica. Este hecho puede hacerte ser capaz de anticipar determinados efectos, así como diversas consecuencias que tu enfermedad pueda tener en ti o en tu vida en general (p. ej. Dejar de hacer cosas que ahora haces, pensar que tu vida va a ser peor de lo que pudiera ser ahora, etc.).

Como en los casos anteriores, nos interesa evaluar esos aspectos, y para ello te presentamos distintas preguntas. Di el grado en que crees que te afectará tu enfermedad A LARGO PLAZO, marcando un número del 1 al 5 . Recuerda que es necesario que contestes a todas las preguntas.

- ¿En qué grado crees que los efectos y consecuencias originadas por la enfermedad te afectarán personalmente, en un futuro?(C.Futuro1)

- ¿En qué grado crees que tu enfermedad afectará a tu familia? (C.Futuro2)

- ¿Y en tu trabajo? (C.Futuro3)

- ;Y en tus relaciones sociales en general? (C.Futuro4)

ESCALA DE RESPUESTA: No afectará nada (1) - Afectará mucho (5)

\section{ESCALA CONTROL-CURA}

Antes te hemos preguntado por las consecuencias que tiene para ti tu Diabetes .También hemos hablado del control y de cómo esa forma de controlar la enfermedad (pincharse, comer a determinadas horas, etc ) puede afectar a tu vida o puede tener determinado impacto sobre ti y tu entorno.

Ahora vamos a preguntarte también por algo relacionado con el control, pero en este caso únicamente queremos que nos indiques lo controlable que te parece que es tu enfermedad, es decir, nos queremos referir a si crees que existen estrategias que permitan o que hagan posible mantenerla bajo control, estable, manejada, etc. No hace falta que pienses en quién debe poner en marcha estas estrategias, o si cuando las ha puesto en marcha, han funcionado. Sólo queremos que pienses en qué grado es posible controlar la enfermedad.

Para indicar todo esto se te van a presentar una serie de preguntas, a las que deberás responder rodeando con un círculo un número del 1 al 5 igual que en ocasiones anteriores (sabiendo que 5 significa que es muy controlable y 1 que no es nada controlable)

- De 1 a 5, ¿en qué grado crees que tu enfermedad es controlable? (ContEnf)

- Y los síntomas con que se manifiesta tu enfermedad, ¿en qué grado crees que pueden controlarse (ContSint)

ESCALA DE RESPUESTA: Nada controlable/s (1)-Muy controlable/s (5)

Ahora queremos que pienses en lo que TU puedes hacer para controlar la enfermedad Es decir nos interesa conocer tu opinión sobre el grado en que crees que tu enfermedad o diversos aspectos de ésta DEPENDEN de lo que TÚ PERSONALMENTE hagas, es decir, están bajo tu control (por ejemplo, hacer ejercicio, llevar una dieta saludable, hacerse análisis continuos, tomarse las cosas con calma, etc.)

Para ello, se te van a presentar una serie de preguntas que, como anteriormente, deberás responder rodeando con un círculo el número del 1 al 5 que represente mejor tu opinión. Recuerda no dejar ninguna pregunta sin contestar.

- De 1 a 5, ¿en qué grado crees que el control de tu enfermedad depende de lo que hagas? (ContPers1)

- Y el manejo de los síntomas que aparecen diariamente, ¿en qué grado depende de tí? (ContPers2)

- Como ya nos indicaste anteriormente, conoces en alguna medida las consecuencias que provoca o puede provocar tu enfermedad. ¿En qué grado crees que puedes controlarlas? (ContPers3)

- Como sabes, los síntomas de tu enfermedad son provocados por diversos factores (p. ej. el estrés, alteraciones emocionales, alimentación, etc.). De 1 a 5, indica en qué grado puedes hacer algo para controlar algunos de esos factores (ContPers4)

ESCALA DE RESPUESTA: No depende (1) - Depende Totalmente (5)

Como sabes, tu diabetes requiere un tratamiento médico y conductual concreto (hacer ejercicio, dieta estricta, etc ) que debes seguir y que es fundamental para mantener tu enfermedad bajo control. Sin embargo, sabemos que a veces los síntomas aparecen, o la enfermedad se descontrola, a pesar de que tú estés siguiendo las recomendaciones médicas. Como sabemos que eso puede ocurrir, queremos conocer tu opinión sobre el grado en que crees que tu enfermedad y diversos aspectos de ésta, está bajo control del TRATAMIENTO, es decir, nos interesa saber hasta qué punto crees que el tratamiento SIRVE para manejar o mantener controlada tu diabetes.

Como en los casos anteriores, se presentan una serie de preguntas que debes responder usando las números del 1 al 5 con el sentido explicado anteriormente Recuerda que es necesario no dejar ninguna pregunta sin contestar

- Piensa en cómo la enfermedad se manifiesta diariamente (altibajos, bajadas y subidas de “azúcar”, etc.). De 1 a 5 , ¿en qué grado crees que el tratamiento sirve para controlar la aparición de estos sintomas? (Cont Tto1)

- Como sabes, muchos de esos altibajos a los que nos referimos pueden estar motivados por causas como el estrés, la ansiedad, emociones como la tristeza, el enfado, etc. También decíamos antes que el tratamiento incluye aspectos médicos, pero también recomendaciones relativas a tu comportamiento (por ejemplo, debe tener unos hábitos de vida saludable, hacer ejercicio físico, llevar una dieta estricta, 
etc.). De 1 a 5 , ¿hasta qué punto crees que estas recomendaciones sirven para controlar esos factores que pueden dar lugar a los altibajos? (Cont'Tto2)

- Además de los síntomas diarios que seguramente padeces, sabes que tu enfermedad puede provocarte algunos trastornos físicos en el futuro. De 1 a 5, ¿hasta qué punto crees que las recomendaciones relativas a tu comportamiento (ejercicio físico, dieta adecuada, etc ) te podrían servir para paliar o reducir dichos trastornos en un futuro? (ContTto3)

ESCALA DE RESPUESTA: No sirve en absoluto (1)-Sirve totalmente (5)

Ahora nos interesa que pienses en las personas que de alguna manera te ayudan a sobrellevar tu enfermedad (médico, cuidador, familia, etc.). Sabemos que muchas veces son estas personas, las que te ayudan de alguna manera a controlar la enfermedad (por ejemplo, tu pareja puede cocinar la comida respetando la dieta, o puede animarte a hacerte un análisis, o tu médico puede mostrar interés en cómo estás cada vez que lo ves, prescribiéndote pautas nuevas, educándote en tu enfermedad, etc.). Como ves, estas personas pueden participar en los cuidados cotidianos que necesitas por tu enfermedad. Queremos que nos indiques, en qué medida crees que ESTAS PERSONAS pueden ayudarte a CONTROLAR tu diabetes.

Como en los casos anteriores, se presentarán una serie de preguntas que debes responder usando la escala numérica explicada anteriormente. Recuerda que es necesario no dejar ninguna pregunta sin contestar.

- De 1 a 5, ¿hasta qué punto crees que otras personas te ayudan a controlar de alguna manera los sintomas diarios de tu enfermedad (p.e. animándote a hacerte un análisis, cocinar la comida respetando tu dieta, etc.? (ContOtro1)

- Como sabes, gran parte de ese malestar diario al que nos referimos puede haber sido provocado, o estar precedido de situaciones que te han alterado de alguna manera (el estrés, alguna discusión, estado de ánimo decaído, que hayas comido algo inadecuado, etc.). De 1 a 5 , ¿en qué grado crees que otras personas pueden ayudarte a controlar esas situaciones que pueden dar lugar a la aparición de síntomas? (ContOtro2)

ESCALA DE RESPUESTA: No ayudan en absoluto (1)-Ayudan totalmente (5)

Finalmente, queremos saber tu opinión acerca del grado en que crees que tu propia diabetes, no la diabetes en general, es curable, es decir, se puede solucionar. Utiliza para contestar los números del 1 al 5 que aparecen junto a la pregunta.

- De 1 a 5 , ¿en qué grado crees que tu enfermedad se puede curar? (Cura)

ESCALA DE RESPUESTA: No se puede curar (1)- Se puede curar (5)

\section{ESCALA CURSO}

Nos interesa que nos digas lo que piensas sobre la duración de tu Diabetes, es decir, nos interesa conocer si crees que la diabetes va a durar poco, o si crees que estará presente durante mucho tiempo. Para que nos lo indiques, te vamos a presentar tres frases. Deberás decirnos cuál de las dos representa mejor tu opinión marcando con una cruz SOLO UNA DE ELLAS.

\section{$\square$ Mi enfermedad estará siempre presente $\square$ Mi enfermedad será pasajera $\square$ ienfermedad es imprevisible (Duración)}

También nos interesa saber tu opinión sobre cómo crees que va a evolucionar la enfermedad, así como los síntomas, conforme pase el tiempo, es decir, queremos que nos indiques si piensas que va a mantenerse estable, si crees que irá evolucionando a mejor, que irá empeorando, etc. Haz como en el caso anterior, marca SOLO UNA DE LAS OPCIONES

$\square$ Mi enfermedad se mantendrá siempre como hasta abora

$\square$ Mi enfermedad empeorará progresivamente

Mi enfermedad mejorará con el tiempo i enfermedad irá cambiando por temporadas(Evolución) 